Sección Dos: Textos

Retos y Experiencias en la Construcción de Paz

\title{
Reflexiones de la educación para la paz en tiempos de postconflicto en Colombia ${ }^{1}$
}

Reflections on education for peace in post-conflict times in Colombia

\author{
Edward Amorocho Herrera \\ eduar333@hotmail.com \\ María Mercedes Giraldo Álvarez \\ maria.giral@gmail.com \\ Jhon Granados Rico \\ jhongranadosrico@gmail.com \\ Carolina Montagut Orozco \\ caromontagut@gmail.com \\ Mónica Hilarión Madariaga \\ mhilarion23@gmail.com \\ Rosa Ludy Arias \\ rarias@javeriana.edu.co \\ Universidad Javeriana Colombia
}

\section{Resumen}

Este artículo tiene como propósito presentar algunas reflexiones sobre los aspectos que se pueden tener en cuenta para la implementación de la Cátedra de la Paz, a partir de las voces y experiencias de notables educadores e investigadores, cuya experiencia se ha desarrollado en torno a este campo temático en Colombia durante los últimos años. Para tal efecto, se abordan los fines de la educación para la paz; los ejes temáticos de la cátedra de la paz- ético moral, jurídico político, socio-cultural, psicosocial y pedagógico; y, finalmente algunas recomendaciones de cómo los docentes deben trabajarla en las instituciones educativas en los niveles de básica y media.

\begin{abstract}
This article aims to present some reflections on the aspects that can be taken into account for the implementation of the Chair of Peace, based on the voices and experiences of outstanding educators and researchers, whose experience has been developed around this field Thematic in Colombia during the last years. To this end, the aims of education for peace are addressed;
\end{abstract}

${ }^{1}$ Recibido: 08/07/2018 Evaluado: 08/08/2018 Aceptado: 20/09/2018 
the thematic axes of the chair of peace - ethical moral, political juridical, socio-cultural, psychosocial and pedagogical; and finally some recommendations on how teachers should work in educational institutions at the basic and middle levels.

Palabras clave: Educación para la paz, Cátedra de la paz, Cultura de paz y Postconflicto.

Keywords: Education of the peace; Chair peace; culture of the peace and postconflict.

\section{Introducción}

Las entrevistas a los expertos fueron realizadas entre el mes de noviembre del año 2014 y el mes de marzo del año 2015. Para la selección de los informantes se tuvo en cuenta criterios como: su participación en procesos de construcción de paz; en experiencias de educación para la paz (cátedra de la paz) y conocimiento en políticas públicas en educación para la paz. Se buscó con estos perfiles enriquecer el texto de tesis de grado, no solo desde el contexto del saber; sino además desde el campo experiencial.

El presente artículo se estructura en tres momentos: el primero, se relaciona con algunas ideas enfocadas a la educación para la paz en tiempos de postconflicto en Colombia, a partir de las experiencias de los investigadores consultados. Segundo, se abordaron los ejes temáticos de la Cátedra de la paz, los cuales son: Ético, Jurídico- Político, Sico social, Socio-cultural y Pedagógico. Y, tercero, algunas recomendaciones a los docentes para la implementación de la Cátedra de la Paz en la escuela.

A continuación, aparecen las ideas de educación para la paz que emergen de las experiencias y voces de los consultados (que se consignan numerados por orden en "E", y cuyo resumen se referencia al final del presente trabajo), de la siguiente manera:

\section{Idea 1: Una educación que sensibilice a los niños con la paz y les enseñe a afrontar democráticamente los conflictos}

\section{E1: Felipe Piedrahita, considera que:}

La educación de paz debe estar enfocada en el aspecto de la sensibilización, para que no suceda, como en el caso de la formación constitucional en donde se replica a los niños como robots que obedezcan la norma. Hablamos entonces de una educación para la paz que presupone el contexto del postconflicto armado y que debe apostarle a crear una cultura de la paz integral, a prevenir la violencia, teniendo como referente o los aportes que en este sentido ha realizado la UNESCO desde su creación en 1992 ( F. Piedrahita, comunicación personal, 11 de mayo 2015). 


\section{Idea 2: Una educación que permita mejorar las relaciones hacia una convivencia pacífica ciudadana}

E2: La experiencia de educación para la paz de Enrique Chaux, inicia con la creación de un grupo de investigación en la Universidad de los Andes en el año 2001, en el cual trabajó Educación para la convivencia, Educación para la paz y Prevención de la violencia; de este trabajo posteriormente nace el programa "Aulas en Paz", el cual en la actualidad se ha implementado en más de cien colegios. En su criterio, la educación para la paz debe tener dos objetivos uno:

Tiene que ver con mejorar el clima de relaciones en el contexto escolar, que a su vez favorecería el aprendizaje en todas la áreas académicas y el segundo, es un objetivo para que los estudiantes desarrollen capacidades para que en las relaciones en su vida cotidiana, en sus relaciones laborales, en sus relaciones románticas, relaciones de amistad, y relaciones con desconocidos, pueda haber mucha más convivencia pacífica (E. Chaux, comunicación personal, 19 de mayo 2015).

A este respecto, el autor hace énfasis en que:

Las competencias ciudadanas tienen en sí mismas unas estrategias pedagógicas, esto es, la capacidad de saber hacer en ciertos contextos y para aprender esas capacidades hay que tener oportunidades para la práctica, es decir aqui el principio más importante es aprender haciendo. Entonces, gran parte de nuestras estrategias pedagógicas están pensadas en crear oportunidades para poner en práctica las competencias que queremos desarrollar, si queremos desarrollar empatía, entonces en vez de decirle a los estudiantes, ustedes tienen que ser empáticos, el propósito es crear oportunidades para que sean empáticos; entonces por ejemplo, leer un cuento y parar la historia en cierto momento y decirles ¿conocen a alguien que pueda estar sintiendo lo que está sintiendo esta persona que estamos leyendo?, ¿Cómo se estará sintiendo esa persona que ustedes conocen?, si ustedes fueran a decirle algo ¿qué le dirían?, si ustedes fueran a escribirle una carta ¿cómo sería esa carta?, escríbanle la carta ya mismo y entonces ahí los ponemos a que en la misma actividad, en el mismo momento tengan que ponerse en los zapatos de otros y pensar cómo está sintiéndose esa persona, cómo está sufriendo una situación y estamos básicamente colocándolos en posición de poner en práctica la empatía allí mismo (E. Chaux, comunicación personal, 19 de mayo 2015).

Idea 3: Una educación para la paz que profundice en las causas del conflicto y la violencia y genere el sentido de corresponsabilidad

E3: Rafael Pabón afirma que:

Hay como tres tipos de orientaciones curriculares sobre estos temas: unos esfuerzos orientados a que los niños discutan los temas referidos al conflicto, para que 
comprendan y lean el conflicto armado; otros esfuerzos buscan que los niños entiendan lo que es el movimiento por la paz, el pacifismo, digamos la historia de los movimientos relacionados con la paz como una posición filosófica, política y social; bajo esta perspectiva, los niños estudian los conflictos de otros países; otros profesores entienden que los niños tienen que entender el conflicto armado colombiano y en este sentido, es un trabajo sobre las causas del conflicto y la violencia. Así mismo, plantea que los objetivos de la educación para la paz, en el marco de este proceso de transición del conflicto al posconflicto en nuestro país debería cumplir tres objetivos, el primero, que los niños entendieran las causas de los conflictos; el segundo, que entendieran de alguna manera el propio conflicto que estamos viviendo y; tercero, que de alguna manera entiendan que el pacifismo es una opción que no es inacción, sino que es activa" (R. Pabón, comunicación personal, 22 de mayo 2105).

En esta misma línea E4: Francisco de Roux señala que:

Otro elemento trascendental a tener en cuenta es la responsabilidad colectiva, tiene que ver mucho con la parte ética, lo que ha pasado en el país es responsabilidad de todos nosotros y todas las generaciones, aqui nadie tiene las manos limpias, tanto por lo que hemos hecho, como por lo que hemos dejado de hacer, por eso lo complicado para la justicia en este momento ¿quién puede tirar la primera piedra? cuando todos estamos involucrados, es muy importante que los estudiantes capten el sentido de la responsabilidad colectiva, porque es lo que nos ayuda después a pensar en la posibilidad de una justicia restaurativa que nos restaure a todos, no solamente a guerrilleros, militares y paramilitares; sino también a empresarios, políticos, papás y escuela ( F. Roux, comunicación personal 2 de junio 2015).

\section{Idea 4: Una educación para la paz que permita desaprender la cultura de violencia y generar confianza y cooperación}

E5: La educadora Marina Caireta, considera como retos esenciales para nuestro país: trabajar por conseguir una paz estructural y desaprender la cultura de la violencia para generar confianza y trabajo en equipo, así mismo expresa que la cátedra debe tener tanto una asignatura específica y ser trabajada transversalmente en todo el currículo de la escuela.

Esta investigadora subraya en relación con la implementación de la Cátedra, lo siguiente:

Para mí la asignatura tiene una parte muy interesante, porque permite que haya un currículo explícito, que se hable de algunos temas que son importantes y que los espacios estén reconocidos, por ejemplo; los derechos humanos o temas de democracia y los temas relacionados con el concepto de paz; no obstante lo anterior, existe el riesgo al tener una asignatura especifica de llegar a concluir que ya no se requiere hacer nada más, siendo que es igualmente necesaria la transversalidad en el currículo de la educación para la paz. Por lo tanto, considero que el modelo debería tener ambas posibilidades, una asignatura con un currículo explícito y ser también trabajado el tema transversalmente (M. Caireta, comunicación personal, 2 de junio de 2015). 


\section{Idea 5: Una educación para la paz que contribuya a construir una vida digna y una vida buena con justicia, derechos y memoria}

E6: Marieta Quintero señala que la educación para la paz en el contexto del post-conflicto armado del país debe contribuir al desarrollo de la justicia, a la cultura de los derechos humanos y a la construcción de memoria histórica, individual y colectiva, para que se concreten los ideales de vida buena y vida digna, lo cual no se opone a que puedan realizarse tratamientos diferenciales atendiendo a los impactos de la violencia en distintos lugares del país.

En este sentido, la autora hace énfasis en la construcción de la memoria histórica, para conocer los hechos de violencia en nuestro país y también para que estos hechos no se vuelvan a repetir; yo creo que una manera de no repetir la historia es conocerla”. Además, señala que la:

Cátedra debe estar orientada por el tema de derechos humanos y, otro aspecto que la podría complementar es la ayuda humanitaria. Adicionalmente, otro componente que estaría muy relacionado con la memoria histórica y que yo lo colocaría dentro de este tema de los contenidos, es el de señalar la importancia de los procesos de posconflicto que han existido en el mundo y cuáles han sido las condiciones de investigación que en Colombia se han creado para la comprensión y la detención de los hechos atroces. M. Quintero, comunicación personal, 2 de junio de 2015).

La educación para la paz permite identificar tres grupos de objetivos: los primeros relacionados con la construcción de una paz estructural con justicia y la garantía de los derechos humanos; los segundos relacionados con una paz cultural con los aprendizajes para la convivencia, la prevención de la violencia y la construcción de sentido y corresponsabilidad por una vida digna y buena y finalmente, el tercer grupo incluye fines relacionados con la paz directa que contribuyan a los aprendizajes para la transformación pacífica de los conflictos, la construcción de memoria y la inclusión social de las víctimas y de los victimarios.

Al respecto, Quintero considera que en:

Un proceso de sesenta años de conflicto armado en donde esto no ha entrado en la escuela, en donde en la misma escuela conviven el hijo del victimario con el hijo de la víctima y el hijo del que toma las decisiones como juez o como el representante de la justicia, que de acuerdo con los intereses que tenga, fortalece al uno o fortalece al otro. No podemos ser ingenuos, se ha de considerar que muchas de las masacres fueron realizadas con el apoyo del mismo Estado, entonces no es lo mismo formar para la ciudadanía que formar para la educación de paz. Cuando se forma para la educación de paz, se educa para reconstruir un tejido que ha sido deteriorado y donde hay que reconocer a las víctimas del conflicto y donde se tienen que reconocer asuntos que aún no hemos reconocido. M. Quintero, comunicación personal, 2 de junio de 2015). 


\section{Ejes temáticos que puede abordar la cátedra de la paz}

Los ejes temáticos que los entrevistados consideran que debería contener la Cátedra de la Paz se orientan al desarrollo de unos principios y contenidos que se enmarcan en cinco ámbitos. El primero, se constituye en un componente ético, en donde se inscriben una ética religiosa fundamentada en un componente espiritual, una ética del cuidado, una ética ambiental, los valores éticos y la dignidad humana. El segundo, el jurídico-político en donde se abordan los principios del derecho internacional humanitario y temas como la justicia, democracia y participación, derechos humanos, gobernanza y gobernabilidad, memoria histórica, victimas y reparación. El tercer componente socio-cultural, contempla temas como el territorio, el contexto, la inclusión y el enfoque diferencial. El cuarto, psico-social, el cual aborda temas como la guerra, el miedo, la capacidad de sobreponerse a eventos de horror, el desarrollo de algunas competencias y el clima institucional y finalmente, un componente pedagógico, en donde se abordan tres elementos, uno orientado al desarrollo de los contenidos, otro al aspecto metodológico y por último, la importancia de la formación a los docentes en el proceso de implementación de esta cátedra.

En el ámbito de la primera dimensión, existe un profundo interés por la importancia de la Ética en todos sus aspectos, al respecto De Roux, afirma:

Existe un componente de tipo ético que invitará a pensar en una ética fundamentada en la dignidad humana (Tuvilla, 2004), en una ética religiosa con fundamentación espiritual, además de pensar en tomar una gran conciencia de que el problema colombiano y el problema del conflicto en este País es un problema espiritual antes que cualquier cosa, es una fractura del ser humano entre nosotros muy profunda y entonces hay que recuperar el valor del ser humano, la grandeza de éste. Yo recuperaría lo espiritual y luego le entraría duro a la dignidad, la dignidad no se la debemos a nadie, ni al gobierno, ni al presidente Uribe, ni al presidente Santos, ni al ejército, la dignidad no se la debemos a la escuela, no se la debemos a la Universidad, la tenemos simplemente por ser seres humanos y es igual para todos los colombianos, es que en Colombia esas diferencias son horribles (F. Roux, comunicación personal, 2 de junio de 2015).

Así mismo, Piedrahita agrega a este componente que hay cosmovisiones éticas, "se está lidiando con un problema ético (Comins, 20003, p.2), valdría la pena preguntarse ¿cómo se está formando a los estudiantes en valores éticos? ¿Qué valores se incluyen en esta formación de cultura de paz y cuáles no hacen parte? la formación ética ya es una formación en cultura de paz" y Marieta Quintero añade que dentro de los contenidos de la cátedra se deben trabajar dos conceptos claves el de ética del cuidado y ética ambiental.

En el contexto del segundo componente Jurídico político, los principios de justicia, derechos, democracia y participación son de gran importancia dentro de los contenidos programáticos que debe incluir la cátedra de la paz. En este sentido, Quintero propone que la cátedra de paz en la escuela debe ser abordada con base en algunos ejes como: la justicia y derechos humanos, memoria histórica, cultura democrática, ayuda humanitaria y solidaridad, y cooperación. Del mismo modo, Pabón resalta la importancia del tema de la participación 
como fundamental para entender cuáles son las causas de los conflictos; otorgándole mayor fuerza al tema de la democracia y las formas de representación y participación en la escuela, escenario inmediato en donde se va de implementar esta cátedra. Así mismo, De Roux indica que hablar de paz:

Presupone algunos elementos de la justicia restaurativa: Primero, la verdad; Segundo, la reparación de las víctimas ya que ha habido una acción de daño; Tercero, la justicia, ha hay que explicarle al niño, uno negocia la verdad, es decir en el fondo si ustedes nos dicen la verdad nosotros los aceptamos en la vida política. Si ustedes restauran a las víctimas en alguna forma nosotros no permitimos que tengan que pagar los años de cárceles que realmente se merecerían, es una negociación. (F. Roux, comunicación personal, 2 de junio de 2015).

En el tercer componente, se agrupan elementos de carácter Socio cultural, Piedrahita señala que:

Dentro del complejo entramado que involucra la cultura de paz se debe incluir la necesidad de defender universalmente una visión particular de los derechos humanos, la democracia, la convivencia, la tolerancia y la interculturalidad. Adicionalmente, Pabón agrega la tolerancia y la inclusión como práctica educativa es importante, porque gran parte de nuestros problemas es la incapacidad que tenemos de leer nuestros propios contextos, es una desgracia que nuestros niños vivan aislados y fragmentados", esto en coherencia con Galtung quien sostiene que: "el tema de la paz no puede dejar por fuera el tema de la guerra, la posibilidad del perdón y la capacidad de ser solidarios, los cuales son temas que tenemos débiles en nuestra propia vida (Galtung, 2003, p.12)

En el ámbito psicosocial, De Roux plantea que dentro de los retos de la educación para la paz estarían la necesidad de desarrollar en los estudiantes conductas resilientes plantea:

No daría explicaciones filosóficas, éticas, políticas, teológicas, porque se quiebran las explicaciones, pondría ejemplos de seres humanos qué puestos en el límite, cuando no tenían ya nada que perder nos han enseñado que hay cosas en las cuales uno no puede ceder, si puede todavía vivir como ser humano. Utilizaría imágenes por ejemplo la señora del Nogal que le mataron el hijo y la nuera y al otro hijo se lo dejaron parapléjico, las mamás de los falsos positivos que el ejército le mató a los hijos y el otro que llega y se quita la prótesis y la pone sobre la mesa para empezar a dar su testimonio y sin embargo están ahí para decir hay que sacar adelante el país, que no nos podemos dejar vencer (F. Roux, comunicación personal, 2 de junio de 2015).

En esta misma dimensión Chaux afirma que:

La educación para la paz debe enfocarse en la disminución de la agresión en los estudiantes frenando este fenómeno en los contextos escolares y extra escolares, al respecto y por ende, plantea que la propuesta de la cátedra de la paz debe partir del trabajo adelantado sobre Las Competencias Ciudadanas, las cuales ya tienen categorías definidas. Por un lado, están los distintos tipos de competencias: 
emocionales, cognitivas y comunicativa y por otro lado, dentro de esas competencias existen ocho competencias relevantes para promover las relaciones pacíficas: Empatía, Manejo de la Rabia, Toma de Perspectiva, Generación Creativa de Opciones, Consideración de Consecuencias, Escucha Activa, Pensamiento Crítico y Asertividad (E. Chaux, comunicación personal, 19 de mayo 2015).

Por último, en el componente pedagógico de la cátedra de la paz con base en los aportes de los entrevistados, debe ser abordado en tres sentidos: el primero, orientado a los contenidos; el segundo, a las metodologías o estrategias pedagógicas y por último, se enfatiza en la importancia de la formación y el acompañamiento a los docentes en el proceso de construcción e implementación de la cátedra de la paz en las escuelas. En este orden de ideas, Caireta recomienda que los ejes de la cátedra de la paz deben abordarse en dos sentidos; uno hacia los contenidos y el otro con base en el aspecto metodológico. En relación a los contenidos, afirma que se vincula la educación para la paz para aquellas cosas que nos permiten avanzar hacia una paz positiva, por ello es importante trabajar el tema de víctimas y el de reparación. En el sentido metodológico, hablar de educación para la paz, implica hablar de convivencia pacífica, de cómo abordar el conflicto y educar en el conflicto, cómo educar en derechos humanos y cómo educar para la democracia. Con la cátedra de la paz, se aspira a que haya una mirada integral de las perspectivas desde donde se abordan temas como el conflicto social, conflicto armado, memoria, violencia, paz positiva, paz negativa y postconflicto, "aspectos que permiten reflexionar desde el reconocimiento de nuestra historia" (Salamanca, Casas, y Otoya, A.,2009, p.34), la búsqueda en escenarios como la familia, la escuela y la sociedad de construcción de culturas de paz, a través de una educación para la paz, la cual se constituye en el objetivo mismo de la implementación de esta cátedra.

\section{Recomendaciones a los docentes para la implementación de la cátedra de la paz en la escuela.}

El proceso de transición hacia la cátedra de paz en la escuela solo será exitoso en la medida que desde el estamento gubernamental se implementen unas políticas públicas que abstraigan a la escuela de la dinámica de deshumanización y cosificación en la que la han sumergido los parámetros de estandarización y las premisas del lucro económico. Se requiere una escuela sensible en donde se valore la comunidad escolar y se construyan proyectos educativos que no estén determinados ni regulados desde contextos externos globalizantes; sino desde los propios protagonistas en donde se preserven y se tengan en cuenta sus realidades, intereses y necesidades de los mismos.

Desde esta perspectiva, el contexto educativo actual tiene un papel protagónico en la construcción de escenarios de paz; por tanto, se hace necesario que la labor de la escuela se centre en la búsqueda de una paz integral que contribuya a la prevención de la violencia social cotidiana, la violencia al interior de la vida familiar y la violencia en la escuela. Igualmente, se requiere de una educación que posibilite el reconocimiento de las causas y las formas de la violencia, comprendiendo que la violencia estructural y cultural y directa que ha generado incluso más víctimas que las que han dejado las confrontaciones armadas del país; a su vez una educación comprometida con develar las representaciones y derivaciones de la violencia, inquieta por formar una conciencia política en los estudiantes y los maestros para que sean ciudadanos comprometidos con la justicia social y el bien común. 
Es necesario que la escuela trabaje con los niños la comprensión de la guerra, como algo próximo a cada uno de nosotros, que ha estado allí cercano, y los efectos que ésta ha tenido a lo largo de la historia de Colombia. Aquí es importante hacer énfasis en la responsabilidad social y cómo lograr desterrar las actitudes de indiferencia que de uno u otro modo hemos asumido todos frente al horror y la barbarie cometida por los distintos actores armados del país, esto además conllevaría a plantear una educación que defienda la necesidad del estudio de la memoria, que visibilice, y reconozca a las víctimas, enfatizando en la no repetición de las acciones atroces, y haciendo posible el rompimiento los ciclos de violencia. De allí surge otro reto para la escuela encaminado a crear y fortalecer en sus estudiantes actitudes de resiliencia que permitan opciones esperanzadoras aun en los contextos y situaciones más adversas. Esto conlleva una escuela en donde se reconoce al otro y se le motiva a seguir adelante desde una perspectiva positiva, esto es, hacia el logro de metas personales, una escuela capaz de volver oportunidades las dificultades y apostarle a la reconstrucción de los sueños.

Resulta evidente que en la dinámica por la búsqueda de una paz directa en la escuela se debe fortalecer una concepción integral de la gestión del conflicto, que incluya, la exploración de mecanismos alternativos de transformación de conflictos, la construcción de rutas que garanticen rigurosidad en los debidos procesos y la implementación de estrategias de abordaje desde categorías como: verdad, memoria, perdón, reconciliación y medidas de justicia restaurativa desde un enfoque preventivo y pedagógico.

Ahora bien, en el plano cultural la escuela debe promover espacios basados en argumentos, posturas críticas y acciones que permitan cuestionar la validez de algunas prácticas que a lo largo de la historia han contribuido a perpetuar los ciclos de violencia, y de otra parte debe fortalecer aquellos legados principios y valores culturales que sirven de patrón para la regulación social y coadyuvan a la construcción de una convivencia armónica, en donde todos sin excepción alguna tienen igualdad de oportunidades y derechos. La educación para la paz requiere de una escuela flexible y creativa en la cual se dé espacio a nuevas miradas y formas de comprender la realidad. Entonces, se trata de una paz que incluya las éticas de la espiritualidad, del cuidado, de la compasión y de la responsabilidad, las cuales privilegien los valores de la tolerancia como: respeto activo, el respeto a las diferencias, la garantía de derechos y la consolidación la democracia y el gobierno escolar.

Finalmente, educar para la paz en tiempo de postconflicto trae como consecuencia repensar el lugar que la sociedad actual le da a quienes ejercen la noble labor de la enseñanza, desde el sagrado encargo de cumplir la premisa de ser formadores de ciudadanos para la paz, y gestores de una cultura de paz. Cumplir efectivamente este legado implica que los educadores reciban una formación seria y rigurosa en el tema, acompañamiento por parte del Estado y las universidades, un reconocimiento profundo que se haga visible en la garantía de condiciones de dignidad y bienestar, respeto y admiración por su hacer político y social y su trascendencia en los procesos educativos, condición sin la cual, la esperanza del logro de una paz integral se quedaría solo en un ideal a alcanzar. 


\section{Entrevistados}

Andrés Felipe Piedrahita: Especialista en Filosofía Política y Magíster en Ciencia Política de la Universidad de Antioquia (Colombia). Docente e investigador de esta Universidad. Actualmente titular de la Cátedra UNESCO en Resolución de Conflictos y Construcción de Paz que se coordina desde la Universidad de Antioquia. Entrevista realizada el 8 de octubre de 2014. Entrevista realizada el 10 de diciembre de 2014.

Enrique Chaux: Doctor en Desarrollo Humano y Psicología de la Harvard University, con Maestría en Sistemas Cognitivos y Neuronales de la Boston University, Profesor Asociado del Departamento de Psicología de la Universidad de los Andes. Fue el coordinador del equipo que construyó los Estándares Colombianos de Competencias Ciudadanas para el Ministerio de Educación Nacional. Ha liderado diversas investigaciones y proyectos relacionados con el desarrollo de la agresión, la intimidación escolar (bullying), la prevención de la violencia y la educación para la convivencia. Lideró la creación y evaluación del Programa Multi-componente Aulas en Paz para la prevención de la agresión y la promoción de la convivencia. Asesoró al Ministerio de Educación en la elaboración de la reglamentación de la Ley 1620 para la Convivencia Escolar. Es autor de múltiples publicaciones, entre ellas el reciente libro "Educación, Convivencia y Agresión Escolar". Entrevista realizada el 27 de noviembre de 2014.

Marina Caireta: Investigadora y docente de la Universidad Autónoma de Barcelona, forma parte del programa de la Escola de Cultura de Pau. Ha trabajado en diferentes programas y proyectos de educación para la paz tanto en el ámbito formal y no formal, realizando actividades dentro y fuera de Cataluña, entre ellos Colombia. Ha publicado distintos artículos en revistas y el libro Juegos de Paz. Entrevista realizada el 13 de marzo de 2015.

Marieta Quintero: Graduada en Lenguas y con maestrías en Filosofía y en Educación, hizo sus estudios doctorales y su post doctorado en ciencias sociales con énfasis en infancia y juventud. Es especialista de la Didáctica de la Literatura Infantil. Docente universitaria. Se ha desempeñado como investigadora en las líneas de investigación educativa, desarrollo humano y educación, perspectivas políticas, éticas y morales de la niñez y la juventud y formación ética y política y principalmente ha trabajado el tema del conflicto armado. Hace parte del equipo que coordina la creación de la Maestría de Paz en la Universidad Distrital. Entrevista realizada el 20 de marzo de 2015.

Rafael Pabón: Docente e investigador del sector educativo. Actualmente es el Coordinador del Programa de Fortalecimiento y Formación de Organizaciones Juveniles en la Fundación CINEP, fue Investigador del Programa de Educación y Políticas Públicas del IDEP. Coautor en el 2005 de la publicación del Cinep: "Biopedagogía: Sistematización de la estrategia educativa del Programa Desarrollo y Paz del Magdalena Medio. El Magdalena Medio, una construcción histórica”. Entrevista realizada el 18 de noviembre de 2014. 


\section{Referencias}

Comins, Irene (2003). La ética del cuidado como educación para la paz (Tesis de Grado). Granada: Universidad de Granada.

Galtung, Johan (2003). Paz por medios Pacíficos. Paz y conflicto, desarrollo y civilización. Bilbao: Red Gernika.

Ley Catedra de la Paz,1732 de 2014 y Decreto 1038 de 2015.

Salamanca, R., Casas, A., y Otoya, A., (2009). Educación para la paz. Experiencias y Metodologías en colegios de Bogotá. Bogotá: PUJ.

Tuvilla Rayo, José (2004). Cultura de Paz. Fundamentos y claves educativas. Editorial Desclée de Brouwer S.A 